University of Nebraska - Lincoln

DigitalCommons@University of Nebraska - Lincoln

Timothy J. Gay Publications

Research Papers in Physics and Astronomy

December 1994

\title{
Energy spread and ion current measurements of several ion sources
}

A. D. Gaus

University of Missouri-Rolla, Rolla, Missouri

W. T. Htwe

University of Missouri-Rolla, Rolla, Missouri

J. A. Brand

University of Missouri-Rolla, Rolla, Missouri

Timothy J. Gay

University of Nebraska - Lincoln, tgay1@unl.edu

M. Schulz

University of Missouri-Rolla, Rolla, Missouri

Follow this and additional works at: https://digitalcommons.unl.edu/physicsgay

Part of the Physics Commons

Gaus, A. D.; Htwe, W. T.; Brand, J. A.; Gay, Timothy J. ; and Schulz, M., "Energy spread and ion current measurements of several ion sources" (1994). Timothy J. Gay Publications. 41.

https://digitalcommons.unl.edu/physicsgay/41

This Article is brought to you for free and open access by the Research Papers in Physics and Astronomy at DigitalCommons@University of Nebraska - Lincoln. It has been accepted for inclusion in Timothy J. Gay Publications by an authorized administrator of DigitalCommons@University of Nebraska - Lincoln. 


\title{
Energy spread and ion current measurements of several ion sources
}

\author{
A. D. Gaus, W. T. Htwe, J. A. Brand, T. J. Gay, and M. Schulz \\ Physics Department and Laboratory for Atomic and Molecular Research, University of Missouri-Rolla, \\ Rolla, Missouri 65401
}

(Received 21 January 1994; accepted for publication 30 August 1994)

\begin{abstract}
Energy spread and extracted current measurements are presented for five different types of ion sources: hot cathode, cold cathode, magnetron, microwave, and electron cyclotron resonance (ECR). The measurements were performed with a $45^{\circ}$ parallel-plate energy analyzer with a resolution of better than $0.5 \mathrm{eV}$ at $500 \mathrm{eV}$ incident energy. Energy spreads ranged from 0.5 to $4 \mathrm{eV}$, while total extracted beam currents ranged from 2 to $30 \mu \mathrm{A}$. Beam currents due specifically to protons, ${ }^{3} \mathrm{He}^{++}$ ions, and $\mathrm{H}_{2}^{+}$ions are also presented. The ECR and magnetron sources gave the best overall performance. 'These, along with the energy analyzer, are currently being used in experiments to study ion-atom collision physics. 1994 American Institute of Physics.
\end{abstract}

\section{INTRODUCTION}

Ion sources play an essential role for accelerator based research, as well as for many industrial and medical applications. Typical requirements arc for large beam intensities, small beam divergence, and high stability. Our application, ion energy-loss spectroscopy, which has proven to be a very effective tool for studying ion-atom collisions, ${ }^{1}$ also requires an ion beam with a very small energy spread. Because the energy spread is crucial for our experiments, we have made systematic measurements of this parameter for a variety of ion sources. In this article we present the first comprehensive energy spread measurements made for five types of ion sources.

The ion energy-loss spectrometer (IELS) at the University of Missouri in Rolla is used to study ion-atom collisions by measuring the energy loss and scattering angle of a projectile ion after collision with a gas target. With this apparatus, the energy loss can be measured with a resolution of better than $1 \mathrm{eV}$ and the scattering angle with a resolution of better than $0.15 \mathrm{mrad}$. This high resolution spectroscopy imposes stringent requirements on the ion source that can be used. The primary ions used on the IELS are protons, ${ }^{3} \mathrm{He}^{++}$ ions, and $\mathrm{H}_{2}^{+}$ions. The original hot cathode source from Colutron Research ${ }^{2}$ had previously been sufficient for studying processes with relatively large cross sections. However, in upcoming experiments, processes with much smaller cross sections will be studied. Therefore it was necessary to develop a new ion source which could deliver more beam intensity than the Colutron source while maintaining an energy spread of less than $2-3 \mathrm{eV}$. Furthermore, improved stability and a longer lifetime were also required.

In this article, energy spread measurements and ion beam currents for protons, ${ }^{3} \mathrm{He}^{++}$, and $\mathrm{H}_{2}^{+}$are presented for the following five different ion sources: (1) hot cathode, (2) cold cathode, (3) magnetron, (4) Evenson microwave, (5) electron cyclotron resonance (ECR). In order to systematically study the performance of these sources, a 45 parallel plate energy analyzer was designed and built. The analyzer has a resolving power of greater than 2000 , corresponding to an absolute resolution of $0.5 \mathrm{eV}$ at an incident energy of $1000 \mathrm{eV}$.

\section{TECHNICAL DESCRIPTION}

\section{A. Ion sources}

Four ion sources were designed and built for comparison with the original hot cathode source. They were designed to fit on a standard 4-5/8 in. ultrahigh vacuum (UHV) flange. All of the sources including the hot cathode source use a plasma discharge for ion production. An extraction aperture is located at one end of each source, with the extraction electrode $1 \mathrm{~cm}$ downstream. The source and extraction aperture are biased $2000 \mathrm{~V}$ positive with respect to the extraction electrode. Extraction geometries were varied slightly, but did not strongly affect source performance. Descriptions of the design concepts specific to each of the ion sources are given below. Details of source designs and operation can be found in the original publications, thus only brief descriptions are given here.

The hot cathode source ${ }^{3}$ is shown schematically in Fig. 1 with typical operating characteristics listed in Table I. Primary electrons are produced by a thoriated tungsten filament and are accelerated by a low voltage (approximately $80 \mathrm{~V}$ ) towards the anode. These primary electrons ionize the atoms of the source gas, thereby creating secondary electrons. Eventually this process leads to a sufficient number of ions

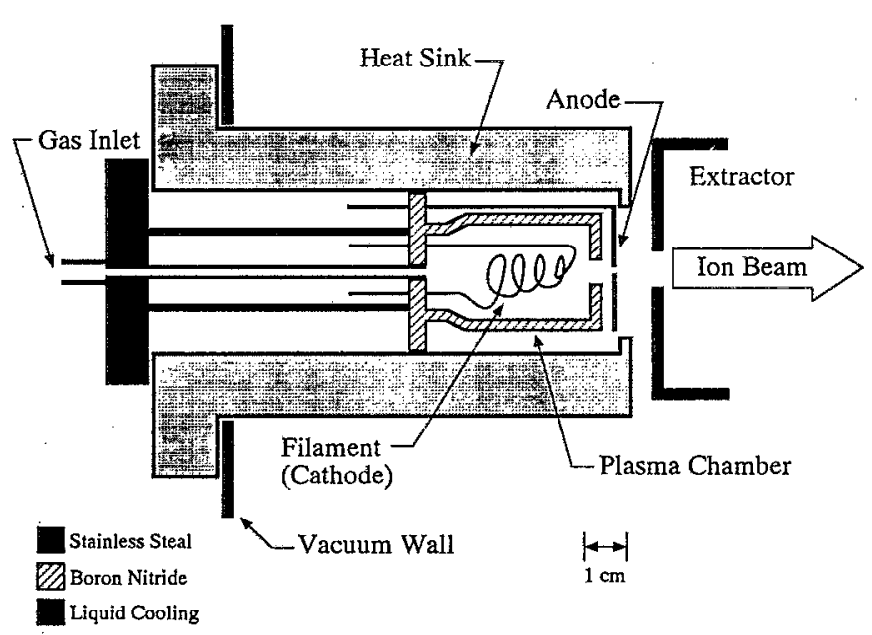

FIG. 1. Schematic diagram of the hot cathode source. 
TABLE I. Operating parameters for the hot cathode source.

\begin{tabular}{ll}
\hline \hline Anode voItage & $60-90 \mathrm{~V}$ \\
Anode current & $100-200 \mathrm{~mA}$ \\
Filament power & $200 \mathrm{~W}$ \\
Extraction aperture diameter & $0.5 \mathrm{~mm}$ \\
Source gas pressure & $100 \mathrm{mTorr}$ \\
Lifetime for producing protons & $<100 \mathrm{~h}$ \\
Lifetime for producing $\mathrm{He}^{++}$ & $<6 \mathrm{~h}$ \\
Stability & Fair \\
\hline \hline
\end{tabular}

and electrons being created, such that a self-sustaining discharge (plasma) is obtained. This source is known to produce ion beams with a very small energy spread (less than $1 \mathrm{eV}$ ), but only moderate ion currents and a short lifetime are realized. The short lifetime is primarily due to bombardment of the filament by ions created in the source. The bias of the source is such that ions are accelerated towards the filament and away from the extraction aperture. This is commonly referred to as "reverse biasing" the source, since the direction of the ion trajectories is opposite that required for maximum extraction efficiency. This reverse bias allows only ions created very near the extraction aperture to exit the source, thus minimizing the overall energy spread.

The cold cathode source is based on a design by Miljevic, ${ }^{4}$ which incorporates a hemispherical stainless steel cathode and an alumina-coated anode. Its schematic and characteristics are given in Fig. 2 and Table II, respectively. The hemispherical geometry of the cathode is such that the electric field accelerates the electrons from the cathode to the center of the anode. This in turn creates an intense region of ionization very close to the extraction aperture, allowing for more efficient extraction of the ions. Because the cathode is not directly heated, very few primary electrons are initially present. Thus, higher voltages and source pressures are required for ignition of the plasma. The present design uses a 2 $\mathrm{cm}$ radius hemisphere, as opposed to Miljevic's $0.5 \mathrm{~cm}$ radius, to increase cathode surface area, which in turn should decrease both the operating voltage and the gas pressure.

The magnetron source is similar to that designed and built by Miljevic ${ }^{5}$ and is shown in Fig. 3 with characteristics listed in Table III. It uses a radial electric field applied between the cylindrical electrodes. This field is perpendicular

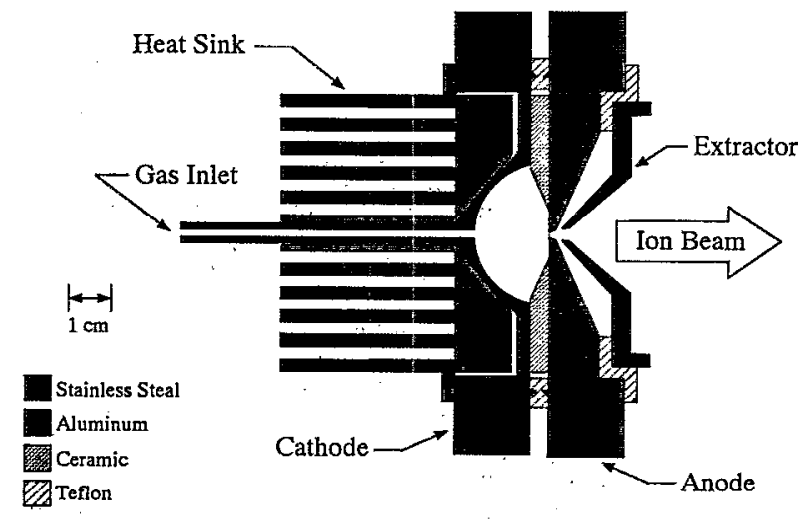

FIG. 2. Schematic diagram of the hemispherical cold cathode source.
TABLE II. Operating parameters for the hemispherical cold cathode source.

\begin{tabular}{ll}
\hline Hemisphere radius & $19 \mathrm{~mm}$ \\
Anode voltage & $300-1000 \mathrm{~V}$ \\
Anode current & $10-50 \mathrm{~mA}$ \\
Extraction aperture diameter & $0.2 \mathrm{~mm}$ \\
Source gas pressure & $400 \mathrm{mTorr}$ \\
Lifetime & Unlimited \\
Stability & Good \\
\hline
\end{tabular}

to an axial magnetic field created by the solenoidal electromagnets, which causes the electrons in the plasma to follow tight helical trajectories, greatly increasing their path lengths. This increase in path length drastically reduces the minimum source gas pressure required to maintain a stable discharge. The intensity of the axial magnetic field was typically set to $400 \mathrm{G}$; however, it was not a critical design parameter.

The microwave source is shown schematically in Fig. 4 with characteristics listed in Table IV. A commercially available Evenson cavity ${ }^{6}$ is used to transmit $2.45 \mathrm{GHz}$ microwave power to the plasma region. The plasma is confined in a thick-walled glass tube approximately $3.5 \mathrm{in}$. long with 0.5 in. o.d. and 0.25 in. i.d. The thick-walled tube was chosen for strength and rigidity only. Microwaves are generated by a power supply with continuously adjustable output power from 0 to $250 \mathrm{~W}$ (typically only $30-50 \mathrm{~W}$ is needed). The microwave power is transmitted via a $50 \Omega$ coaxial cable and is fed into the cavity through a type " $N$ " connector. Impedance matching and cavity tuning arc accomplishcd by using the sliding conductor and tuning stub provided with the cavity. Primary electrons are produced by the use of a tesla coil arc. The primary electrons absorb energy from the microwaves and ionize the atoms in the source gas, thus creating a self-sustaining discharge.

The ECR source is shown schematically in Fig. 5 and its operating parameters are given in Table $\mathrm{V}$. The basic operating principle of an ECR source is similar to that of a standard microwave source in that electrons are heated due to the absorption of power from the microwave field. However, the ECR source uses a magnetic field to produce cyclotron motion of the electrons in the plasma (similar to that of the magnetron source). The cyclotron frequency of the electrons

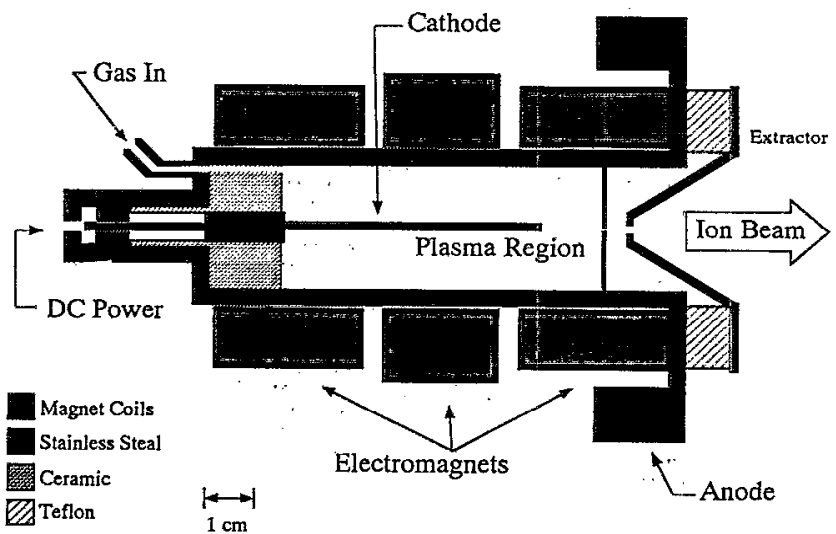


TABLE III. Operating parameters for the magnetron source.

\begin{tabular}{ll}
\hline \hline Anode voltage & $300-1000 \mathrm{~V}$ \\
Anode current & $10-50 \mathrm{~mA}$ \\
Magnet field & $400 \mathrm{G}$ \\
Magnet power & $300 \mathrm{~W}$ \\
Extraction aperture diameter & $0.5 \mathrm{~mm}$ \\
Source gas pressure & $10-100 \mathrm{mTorr}$ \\
Lifetime & $250 \mathrm{~h}$ \\
Stability & Good \\
\hline
\end{tabular}

is directly proportional to the magnetic field intensity, and can be adjusted to match the frequency of the microwave field. When the frequencies match, the cyclotron motion is in resonance with the microwave field so that photons are more efficiently absorbed than without the magnetic field. As a result, the electrons have a much higher energy than in a standard microwave source and thus more intense ionization can occur. This increased ionization produces significantly higher beam intensities and higher charge states than standard microwave sources. The ECR source presented in this article is based on a design used for molecular beam epitaxy studies. ${ }^{7}$ It was slightly modified by placing an extraction aperture and electrode at the exit of the source and by utilizing independently housed magnets for easy adjustment and modification. The plasma is excited by the $2.45 \mathrm{GHz}$ microwaves in resonance with an $875 \mathrm{G}$ magnetic field, which corresponds to a $2.45 \mathrm{GHz}$ cyclotron frequency for electrons. The magnetic field intensity is uniform within the plasma region.

The cavity serves only as a terminator to the coaxial cable, not a resonant cavity. Optimal antenna length was determined empirically to be about $4 \mathrm{~cm}$ long, agreeing closely to the original design. The microwave power delivery system is identical to that used by the Evenson microwave source previously described. Typical microwave power (forward) ranged from 10 to $30 \mathrm{~W}$, with only $2-5 \mathrm{~W}$ reflected. The source could be operated with or without an external impedance matching device. The matching device made it easier to initiate a discharge, but did not greatly enhance overall performance. The magnets consume about $600 \mathrm{~W}$ of power and therefore require liquid cooling.

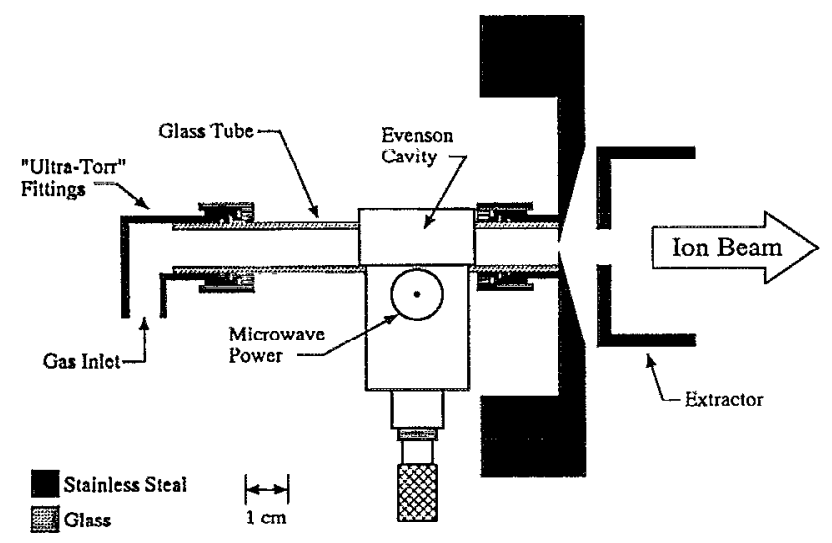

FIG. 4. Schematic diagram of the microwave source.
TABLE IV. Operating parameters for the microwave source.

\begin{tabular}{ll}
\hline Microwave frequency & $2.45 \mathrm{GHz}$ \\
Microwave power & $30-70 \mathrm{~W}$ \\
Source gas pressure & $100 \mathrm{mTorr}$ \\
Extraction aperture diameter & $0.5 \mathrm{~mm}$ \\
Lifetime & $>1000 \mathrm{~h}$ \\
Stability & Good \\
\hline
\end{tabular}

\section{B. Parallel plate energy analyzer}

In order to measure the energy spread of the sources, a new energy analyzer was designed and built, and installed on the "test bench" facility (described below). After testing the sources and the analyzer on the test bench facility, the new analyzer was then installed on the IELS. A 45 parallel-plate design was chosen over other common designs using cylindrical and hemispherical geometries due to its simplicity and ease of construction. Also, the acceptance angle and focusing properties were not considered to be important design criteria since the ion beam is collimated to less than $0.15 \mathrm{mrad}$. The analyzer is shown schematically in Fig. 6 with its performance characteristics listed in Table VI.

The front plate was machined from 3/16-in.-thick aluminum plate, while the backplate was punched from 1/16-in.thick stainless steel. Eleven 1/32-in.-thick stainless steel shim plates (punched) were used around the perimeter of the analyzer to prevent field leakage from the vacuum chamber. The plates required no special surface treatments or preparation such as polishing or etching. The plate assembly is held together by ten nylon $1 / 4-20$ threaded rods with $3 / 8$ in. nylon spacers between each plate. A voltage divider chain is connected to the plates giving $1 \mathrm{M} \Omega$ resistance between each plate and $12 \mathrm{M} \Omega$ resistance overall. The analyzing slits are 0.003 in. wide by approximately $1 / 2$ in. long and were cut using a spark cutter. The distance between the slits is 14.0 in. and the depth of the spectrometer is approximately 4.85 in.

The two most relevant properties of an analyzer are the resolving power, $R$, and the spectrometer constant, $k$. They are defined as

$$
R \equiv \frac{E}{\Delta E}, \quad k \equiv \frac{E}{V}
$$

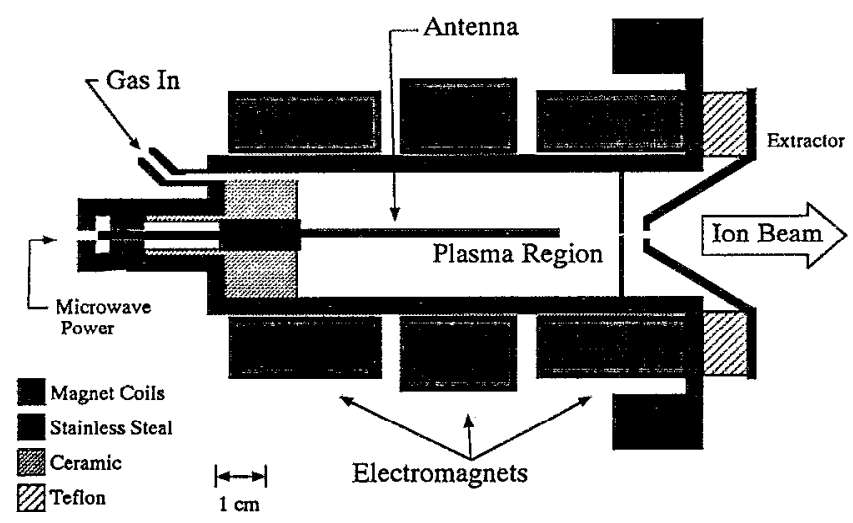

FIG. 5. Schematic diagram of the ECR source. 
TABLE V. Operating parameters for the ECR source.

\begin{tabular}{ll}
\hline Microwave frequency & $2.45 \mathrm{GHz}$ \\
Microwave power & $10-50 \mathrm{~W}$ \\
Magnetic field & $875 \mathrm{G}$ \\
Magnet power & $650 \mathrm{~W}$ \\
Extraction aperture diameter & $0.5 \mathrm{~mm}$ \\
Source gas pressure & $<1 \mathrm{~m}$ Torr \\
Lifetime & Unlimited \\
Stability & Good \\
\hline
\end{tabular}

where $E$ is the energy of the particle, $V$ is the analyzer plate voltage, and $\Delta E$ is the energy resolution. Note that the resolving power is sometimcs cquivalently specified as relative resolution, which is the reciprocal of $R$. These two quantities are determined mainly by the geometry and size of the analyzer. For a 45 parallel plate analyzer

$$
\frac{\Delta E}{E}=\frac{2 w}{l}+2 \alpha^{2}+\beta^{2}, \quad k=\frac{l}{2 d},
$$

where $l$ is the distance between the analyzing slits, $w$ is the width of the slits, $d$ is the distance between the front and backplates, and $\alpha$ and $\beta$ are the acceptance angles of the analyzer in the plane and perpendicular to the plane of dispersion. For conditions under which the beam is not decelerated (as explained below), the values of $2 \alpha^{2}$ and $\beta^{2}$ are negligible $\left(10^{-9}\right)$ compared to $2 w / l\left(10^{-4}\right)$. Only in the case of high deceleration does the divergence of the beam become large enough to contribute to the resolving power of the analyzer. Using the given dimensions, theoretical values of 2330 for the resolving power and $1.45 \mathrm{eV} / \mathrm{V}$ for the spectrometer constant are oblained. At an incident energy of $2000 \mathrm{eV}$, which is the extraction voltage used in the sources, the theoretical absolute resolution is calculated to be $0.86 \mathrm{eV}$.

In order to achieve higher absolute resolution and maintain the $2000 \mathrm{~V}$ source extraction, a five element deceleration lens and an electrostatic quadrupole steering element were inserted in front of the analyzer. The lenses were mounted on an aluminum track using $1 / 4 \mathrm{in}$. alumina rods for alignment. This simple "track" system was utilized to allow precise axial alignment of the deflector, lens, and analyzer without

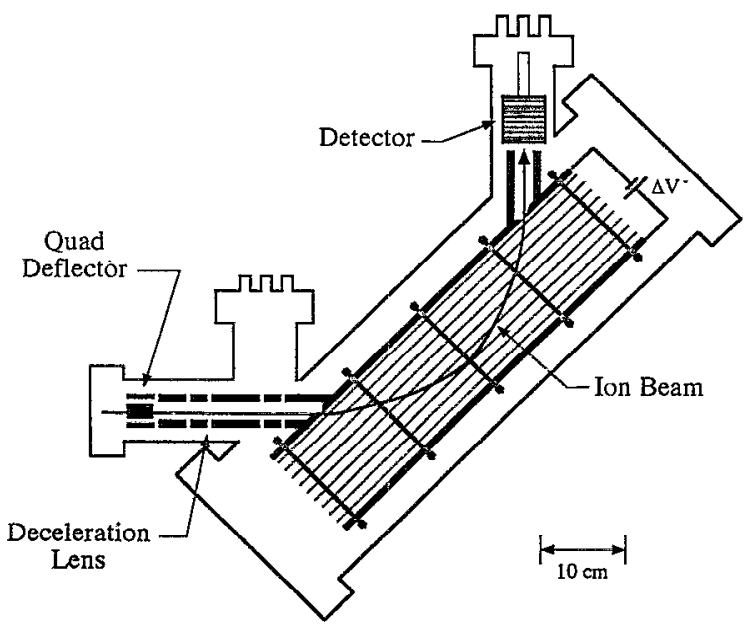

FIG. 6. Schematic diagram of the parallel-plate analyzer.
TABLE VI. Characteristics of the parallel plate energy analyzer.

$\begin{array}{ll}\text { Theoretical resolving power } & 2330 \\ \text { Theoretical resolution at } 1000 \mathrm{eV} & 0.43 \mathrm{eV} \\ \text { Theoretical resolution at } 500 \mathrm{eV} & 0.22 \mathrm{eV} \\ \text { Measured resolution at } 1000 \mathrm{eV} & <0.7 \mathrm{eV} \\ \text { Measured resolution at } 500 \mathrm{eV} & <0.5 \mathrm{eV} \\ \text { Slit width } & 0.0076 \mathrm{~cm}(0.003 \text { in.) } \\ \text { Slit separation } & 35.6 \mathrm{~cm}(14.0 \mathrm{in} .) \\ \text { Plate separation } & 12.3 \mathrm{~cm}(4.85 \text { in.) } \\ \text { Spectrometer constant } & 1.45 \mathrm{eV} / \mathrm{V}\end{array}$

the need of complicated machining techniques. The elements were constructed from 1.5-in. aluminum tubing of $1 / 8$ in. wall thickness. An aluminum tube of the same dimensions was heliarced to the analyzer front plate at $45^{\circ}$ so it could be aligned and attached easily to the lens track.

The entire analyzer and lens assembly was mounted inside an 8-in.-diam by 22-in.-long stainless steel tube with 10 in. UHV flanges on both ends. The tube was then mounted at $45^{\circ}$ with respect to the main beam line by a 4 and $5 / 8 \mathrm{in}$. UHV flange and 3 in. tube. A focused dynode mesh electron multiplier was used as the detector, and was mounted on a 4 and $5 / 8$ in. UHV flange so that it could be replaced easily without moving the analyzer. Neither the analyzer nor the vacuum chamber required any adjustable alignment mechanisms, greatly simplifying both construction and operation.

The analyzer performance was tested by measuring the overall energy resolution of a proton beam from the hot cathode source using $2000 \mathrm{~V}$ for extraction. Using no deceleration, the overall resolution (details of this measurement are explained below) was measured to be about $1 \mathrm{eV}$. Since the hot cathode source was thought to have an energy spread of much less than $1 \mathrm{eV}$, the analyzer resolution was estimated to be about $1 \mathrm{eV}$ for a proton beam of $2000 \mathrm{eV}$ incident energy. Decelerating the beam to $1000 \mathrm{eV}$ incident energy gave an overall resolution of $0.7 \mathrm{eV}$. It was concluded that the energy spread of the hot cathode source may be limiting the resolution of the analyzer. Further measurements confirmed this conclusion, since a lower overall resolution was achieved with the magnetron source at an incident energy of $1000 \mathrm{eV}$.

Calibration of the analyzer was accomplished by decelerating the incident beam, and adjusting the plate voltage until the beam passed through the analyzer and was incident on the detector. By recording the required plate voltage at several incident energies, the spectrometer constant of the analyzer could be unambiguously determined. Using this method, the spectrometer constant proved to be independent of the incident energy and cqual to the theoretical value to within $3 \%$. Some discrepancy to the theoretical value could occur because the spectrometer constant is sensitive to the angle of incidence of the beam with respect to the optic axis of the spectrometer. As a second verification, an energy-loss spectrum of a proton beam passing through a thin argon gas target located between the Wien filter and the analyzer was measured. Since the excitation energies for various argon states are well known, the corresponding peaks in the energy-loss spectrum were used to calibratc the analyzer. From this energy-loss spectrum, the spectrometer constant 


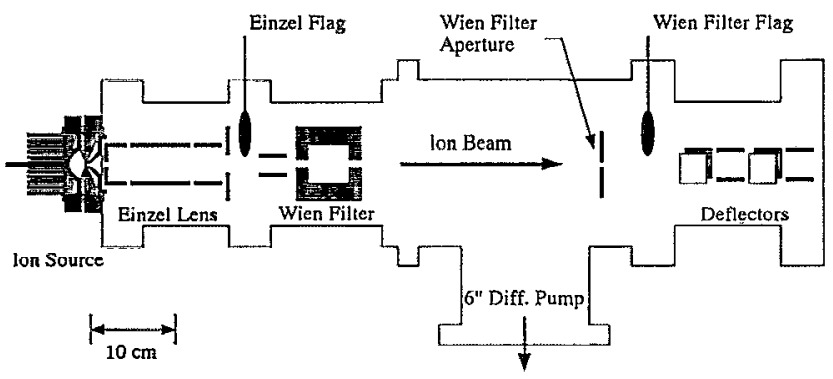

FIG. 7. Schematic diagram of the test bench facility. The energy analyzer is located directly to the right of the deflectors.

was determined to be the same as that obtained from the first method described above.

\section{Measurement apparatus and technique}

A "test bench" facility was used to test the analyzer and subsequently the ion sources. It is shown schematically in Fig. 7. The test bench facility consists of the ion source, Einzel lens, Wien filter, and energy analyzer. The system is pumped by a 6 in. diffusion pump backed by a 10 cubic feet per minute (c.f.m.) rotary vane pump. During typical source operation, the chamber pressure is about $5 \times 10^{-6}$ Torr, which closely matches the vacuum conditions of the source region on the IELS.

The source and its associated electronics are floated to $2000 \mathrm{~V}$ above ground for extraction. An Einzel lens and Wien filter, which immediately follow the source, separate the ion species and allow the desired ions to exit the filter through a $1 \mathrm{~mm}$ aperture located approximately $50 \mathrm{~cm}$ from the source extraction aperture. The Wien filter itself can act as an energy analyzer, but has an energy bandpass of approximately $80 \mathrm{eV}$, thus did not adversely affect the energy spread measurements. Deflectors are used to steer the bcam into the energy analyzer.

Energy analysis was accomplished by setting the analyzer plate voltage to allow a $2000 \mathrm{eV}$ beam to pass through the analyzer and hit the detector. The exact pass voltage is found empirically by tuning the voltage about its theoretical value. The energy spread of the beam was determined by sweeping a -5 to $+5 \mathrm{~V}$ offset on top of the pass voltage and reading the beam intensity incident on the detector. Using the offset voltage as the abscissa and the detcctor output as the ordinate, the energy profile was traced out on an $X-Y$ recorder. A typical profile is shown in Fig. 8. The energy spread was taken to be the full width at half-maximum (FWHM) of this profile. If the spread was less than $2 \mathrm{eV}$, the measurement was repeated using lower incident energies (higher deceleration) until a lower limit in FWHM was reached. Theoretically, the square of the overall measured resolution can be plotted as a function of the square of the incident energy, taking the $y$ intercept as the actual source resolution. However, at deceleration voltages larger than $1000 \mathrm{~V}$, the beam intensities are greatly reduced and the energy spread measurement becomes less reliable. It is assumed that the ultimate resolution of the analyzer is limited by the increasing divergence due to high deceleration of the beam. The terms $2 \alpha^{2}$ and $\beta^{2}$ in Eq. (2) then become a sig-

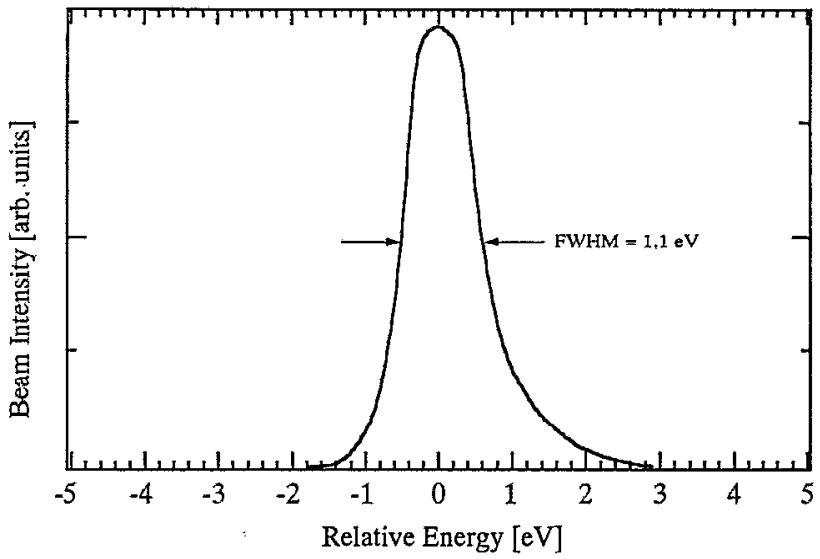

FIG. 8. Typical energy profile measured by the test bench and energy analyzer. The energy spread was taken as the FWHM of the energy profile. The profile shown is of a proton beam from the hot cathode source.

nificant factor in the relative resolution. Therefore, we chose to report the lowest measured value as an upper limit for the source energy spread. In all cases, protons were used to determine the energy spread. These energy spread measurements were repeated several times, and were reproducible to within $0.2 \mathrm{eV}$.

The total extracted ion current was determined by measuring the current on the "Einzel flag" inserted between the Einzel lens and the Wien filter (see Fig. 7). The flag is a flat lantalum disk with no special biasing to prevent secondary emission. The beam currents due specifically to proton, ${ }^{3} \mathrm{He}^{++}$ions, and $\mathrm{H}_{2}^{+}$ions were measured by inserting the "Wien filter flag" just after the Wien filter aperture. This flag is identical to the Einzel flag. The transmission of the Wien filter was much less than 1.0; thus, the measured beam currents should only be used for comparison of source the performances. It must be noted that, in general, total ion current measurements cannot be used as either a reliable or a definitive measure of ion the source performance due to the strong dependence of ion beam intensities on the operational parameters of the source. ${ }^{8}$ The values reported in this article serve only as a comparison of sources operating under very similar extraction conditions and should be used with caution when comparing to sources in other studies or systems.

\section{RESULTS}

The results of the energy spread and ion current measurements are summarized in Table VII. Also listed in Table VII are estimated lifetimes of each source. All the new sources produced specific ion currents that were greater than or equal to those of the hot cathode source. The magnetron source gave the lowest energy spread of $0.5 \mathrm{eV}$, while producing moderate beam currents. In contrast, the ECR source gave the highest ion currents, with a moderate energy spread of $2.2 \mathrm{eV}$. The cold cathode dc source gave a relatively high energy spread compared to most of the other dc sources. This is probably due to the higher anode voltage required for this source. This source also required high gas pressure, and thus appeared to be the least desirable source for the IELS. 
TABLE VII. Results of energy-spread and ion-current measurements. "..." indicates "not available."

\begin{tabular}{lcccccc}
\hline \hline & $\begin{array}{c}\text { Total } \\
\text { current } \\
\text { Ion Source }\end{array}$ & $\begin{array}{c}\text { Proton } \\
\text { current } \\
(\mu \mathrm{A})\end{array}$ & $\begin{array}{c}\text { Alpha } \\
\text { current } \\
(\mathrm{nA})\end{array}$ & $\begin{array}{c}\mathrm{H}_{2}^{+} \\
\text {current } \\
(\mathrm{nA})\end{array}$ & $\begin{array}{c}\text { Energy } \\
\text { spread } \\
(\mathrm{eV})\end{array}$ & $\begin{array}{c}\text { Useful } \\
\text { lifetime }\end{array}$ \\
\hline Hot cathode dc & 25 & 30 & 0.1 & 200 & 0.7 & $100 \mathrm{~h}$ \\
Hemispherical dc & 10 & 20 & 0.0 & 200 & 2 & Unlimited \\
Magnetron dc & 2 & 30 & 1.0 & 100 & 0.5 & $250 \mathrm{~h}$ \\
Microwave & $\cdots$ & 30 & 0.0 & $\cdots$ & 4 & $1000 \mathrm{~h}$ \\
ECR & 30 & 500 & 1.5 & 500 & 2 & Unlimited \\
\hline \hline
\end{tabular}

The Evenson microwave source gave an energy spread of $4 \mathrm{eV}$, and provided proton currents very similar to the hot cathode source. Important features of this source are its long lifetime and simplicity. This source was the least expensive of all sources tested, and is very useful for energy-loss experiments that do not require excellent energy resolution. This source was installed on the IELS in 1990, and has been used to study electron-capture and ionization processes.

For experiments requiring higher ion currents or better energy resolution, the magnetron and ECR sources showed the best performance. Since both sources are physically identical, but operated in two different modes, one can choose the appropriate mode based on particular experimental constraints. For moderate resolution and high currents the source is used in ECR mode. On the other hand for high resolution and moderate currents the source is used in magnetron mode. Of all the sources tested, these were the only ones beside the hot cathode source that were able to produce ${ }^{3} \mathrm{He}^{++}$ions. This is probably due to the relatively low operating source pressures, which significantly lowers the probability of recombination of the ${ }^{3} \mathrm{He}^{++}$ions with the electrons in or around the source. Also, the lower pressures yield longer electron mean free paths between collisions and thus results in higher electrons energies, which in turn increase the ionization probabilities. The hot cathode source could only produce ${ }^{3} \mathrm{He}^{++}$ions for about $4 \mathrm{~h}$ because of increased cathode deterioration at high power. The magnetron and ECR sources did not require higher power for ${ }^{3} \mathrm{He}^{++}$production and therefore did not exhibit any decrease in their useful lifetimes.

The beam current measurement due to specific ion species were very sensitive to all source operating conditions. In fact the ratio of a particular species current to the total current varied from one source to the next. This ratio also varied for each source when source operating parameters were changed (i.e., gas pressure, input power, etc.). Our studies show this variance is primarily a combination of two independent effects. First, the relative production rate of each ion species changes drastically with source parameters. Second, the divergence of the beam is also greatly affected by source parameters. These two effects, when combined, can give unexpected changes in the measured beam current from a specific ion species. By studying measurements of ion current incident directly on the Wien filter aperture, it was concluded that the change in divergence is at least as significant as the change in the production rate of a particular ion species. Because of these effects, one must be careful in using par-

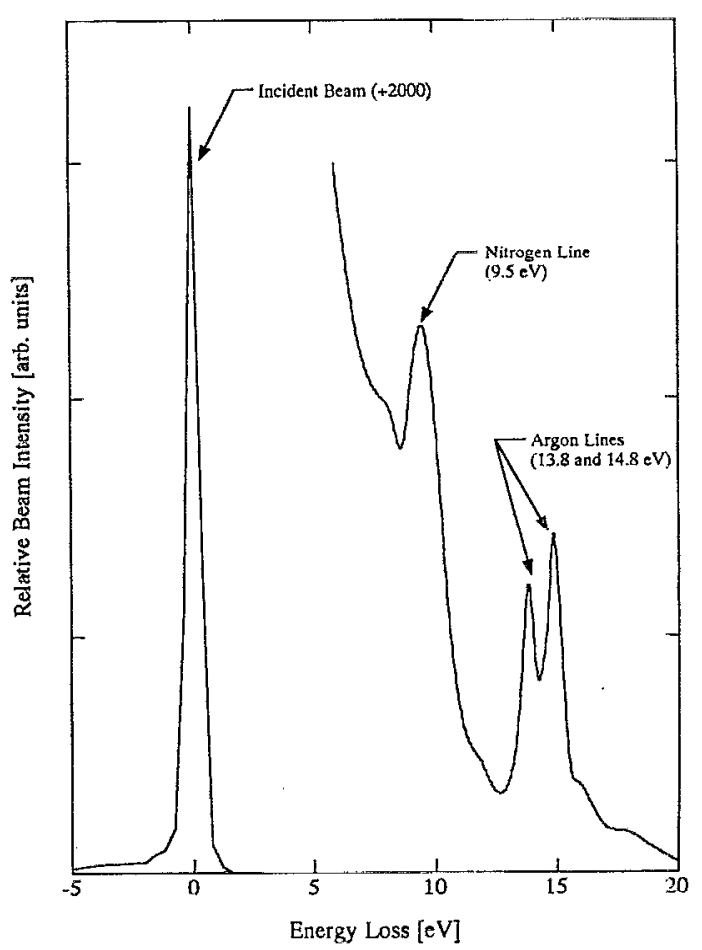

FIG. 9. Energy-loss spectrum of $2 \mathrm{keV}$ protons incident on a thin argon target. The protons were produced by the magnetron source. Well-separated excilation lines of argon are easily identified.

licular ion currents as the only basis for a comparison of sources.

As mentioned above, the analyzer was calibrated by taking an energy-loss spectrum of protons colliding with an argon target. This spectrum was taken using the magnetron source and is shown in Fig. 9. Pronounced peaks corresponding to excited states of argon are readily observed. The peaks at 13.8 and $14.8 \mathrm{eV}$ are due to excitation of the $M$ states of argon. The peaks at lower energy are due to excitation of atoms from background gas. The widths and separation of the argon excitation lines show that both the resolution of the analyzer and the energy spread of the magnetron source are less than $1 \mathrm{eV}$. This spectrum also shows that the source is very suitable for high accuracy energy-loss spectroscopy studies.

\section{CONCLUSION}

Energy spreads and beam intensities for various ion sources have been measured. For low resolution measurements, the microwave source is optimal due to its ease of operation, good stability, and practically unlimited lifetime. For high resolution measurements, the ECR/magnetron combination source is most suitable. Since the ECR and magnetron sources are physically the same, the mode of operation can be chosen for the particular experiment. In ECR mode, this source provides the largest beam intensity while still maintaining a sufficiently low energy spread for most applications. In magnetron mode, this source has an excellent energy spread while maintaining sufficient beam intensities for most applications. 


\section{ACKNOWLEDGMENTS}

The authors wish to express their appreciation to Z. Sitar and R. Davis for the original ECR source plans. This work is supported by the National Science Foundation and the Department of Energy.

${ }^{1}$ J. T. Park, Adv. At. Mol. Phys. 19, 67 (1983).

${ }^{2}$ Colutron Research Corp., Boulder, CO.
${ }^{3} \mathrm{M}$. Menzinger and L. Wahlin, Rev. Sci. Instrum. 40, 102 (1969).

${ }^{4}$ V. Miljevic, Rev. Sci. Instrum. 55, 931 (1984).

${ }^{5}$ V. Miljevic, Rev. Sci. Instrum. 55, 121 (1984).

${ }^{6}$ F. C. Fehsenfeld, K. M. Ėvenson, and H. P. Broida, Rev. Sci. Instrum. 36, 294 (1965):

${ }^{7}$ Z. Sitar, M. J. Paisley, D. K. Smith, and R. F. Davis, Rev. Sci. Instrum. 61, 2407 (1990).

${ }^{8} \mathrm{G}$. D. Alton, Ionization Phenomena and Sources of Ions, Applied Atomic Collision Physics Vol. 4, edited by H. W. Massey (Academic, New York, 1983), Chap. 2. 